\title{
Computer Simulation of Energetic Cluster Impacts on Solid Surfaces
}

\author{
Roger P. Webb \\ Surrey Ion Beam Centre, Advanced Technology Institute \\ University of Surrey, Guildford, GU2 7XH, UK
}

\begin{abstract}
Molecular Dynamics computer simulation is used to demonstrate the behaviour of surfaces upon impact by energetic molecules. At low energies and glancing angles the fullerene molecules can be made to scatter from the surface intact. The coupling of the deposited energy into the surface vibrational modes, particularly for layered materials like HOPG graphite, can lead to what appears to be anomalous behaviour. This is explored and compared with experimental results.

Not all fullerene molecules are spherical. The $\mathrm{C}_{76}$ fullerene is elliptical in shape. Computer simulations are used to investigate the effects of shape on the scattering of molecules from a graphite surface.

Molecular species have been used in ion implantation for doping shallow layers in silicon. There are two contradictory things that can happen when a cluster or molecule is implanted. The molecule will damage the crystal structure with each impact and in so doing could prevent the channelling of the implanted ions, thereby reducing the over-all range of the implantation. It is also possible that the atoms in the "front" of the cluster/molecule will interact with the surface first, pushing aside the surface atoms so that the atoms of the cluster/molecule following behind might not interact with them so strongly and hence be able to penetrate the solid more deeply. This will result in a deeper implantation range profile. Simulations are compared between single atom and molecular species to investigate which of these mechanisms, if any is operating at low implantation energies.

Two clearly observed vibrational modes are excited in a graphite surface by molecular impacts. It is shown that these vibrational modes can assist in the desorption of loosely bound adsorbates from the surface. At higher impact energies it is shown that the surface disruption caused by the impact can both aid and inhibit the desorption process depending upon the position and energy of the initial fullerene impact in relation to the position of the adsorbate. Some simple conclusions about the “desorbing power" of a fullerene impact as a function of energy are drawn.
\end{abstract}

\section{INTRODUCTION}

The interaction of energetic clusters with solid surface is a subject that has been under investigation both experimentally and computationally for more that 30 years. The computer modelling of the interaction of energetic clusters with surfaces is 
bounded on the one hand by the collision of large blocks of material at acoustic velocities and on the other by single ion bombardment. In the former case this can be modelled quite successfully by continuum methods and in the latter by atomistic simulations.

The effects of the impact of an energetic cluster on a solid surface and the fate of the cluster itself depend on many factors. These will include: the velocity of the cluster; the mass of the constituent atoms in the cluster relative to the mass of the target atoms; the number of atoms in the cluster; and the binding of the atoms to each other in the cluster.

The velocity of the cluster will determine whether the cluster will embed itself in the target or bounce away from the surface. At low impacting velocities: the binding energy of the cluster to the solid surface will determine if the cluster succeeds in bouncing free of the surface or remains attached to the surface after impact; and the binding energy of the cluster atoms to each other will determine if the cluster breaksup and shatters on impact or remains intact. If the cluster shatters on impact then the scattering of the resulting constituent pieces of the cluster across the surface can result in the removal of loosely bound debris and particles on the surface as well as the general smoothing of an initially uneven surface.

The relative mass of the constituent atoms of the cluster to the mass of the target will determine at high impact velocities the penetration depth of the constituent particles and the probability of the atoms of the cluster penetrating through the solid in close proximity to each other. The more they remain close to each other the more likely that the behaviour of the cluster impact will behave differently from the impact of the same number of single atom ions one after the other and hence produce a "nonlinear" effect.

The number of atoms in the cluster will determine the total amount of kinetic energy delivered to the surface. The mass ratio of the cluster atoms to target atoms and the initial velocity determines the depth over which this energy is distributed. Hence, the combination of these two factors, allow the energy density to be changed for the same constituent atoms. This can not be done in conventional single atom ion irradiation as the amount of energy being deposited can not be decoupled from the depth over which the energy is deposited, without changing the constituent materials.

The main emphasis here will be to look at the effects of clusters used in scattering, implantation and to induce desorption of large molecules adsorbed on a solid surface.

\section{Computer Simulation Model}

Molecular Dynamics simulations of the sputtering process have been used successfully for more than 40 years ${ }^{1}$ to help understand the complex physical phenomenon that occur after the impact of an energetic particle on a solid surface. There has been a long history using and improving the simulation models to gain greater insight over that time. Since 1992 we have been modelling the interaction of $\mathrm{C}_{60}$ molecules with surfaces ${ }^{2,3}$. By careful comparisons with experiments, performed in collaboration with the University of Karlsruhe, the simulation models have been verified for both low energy scattering of the molecules ${ }^{4}$ and the surface damage created from the impact ${ }^{5}$. The model has been used to investigate how the deformation 
of the surface caused by the impact of a large molecule such as $\mathrm{C}_{60}$ can cause the ejection of loosely bound molecules from the surface ${ }^{6,7}$. Most recently it has been shown computationally how the deformation of a silicon surface caused by a high energy $\mathrm{C}_{60}$ impact can eject a large polystyrene molecule without fragmentation of the polystyrene molecule ${ }^{8}$. This is of particular interest to the Secondary Ion Mass Spectrometry community who are starting to use molecular - largely $\mathrm{C}_{60}, \mathrm{SF}_{5}$ and $\mathrm{Au}_{3}$ - and cluster beams as sputtering beams because of their ability to produce high sputtering yields without causing damage deep in the target. They are also very attractive to use as they produce a much higher fraction of intact large molecular species instead of the normal fragments when sputtering organic films. Understanding the intact molecular ejection mechanisms is fundamental to optimising the yield of such molecules. Simulations have shown that the original concept that the impacting $\mathrm{C}_{60}$ shatters on impact and scatters across the surface causing substantial molecular ejection is incorrect. The simulations make it quite clear that the fullerene impact causes co-ordinated motion in the target material which propagates out from the impact site and lifts any surface molecules, pushing them gently away from the surface, this can occur with sufficient energy transfer to gently eject the molecule intact from the surface. This simulation study is still very much in the early stages. A more detailed analysis of the behaviour of the co-ordinated motion with respect to the impact parameters of energy, angle, cluster size, target material and structure could provide useful input into an experimental programme. The work reported here forms the first part of a more detailed study of the behaviour of adsorbed molecules close to the impact point of an energetic cluster.

The simulation model employed in this study has been described in detail in the past $^{9}$. In brief a Molecular Dynamics scheme is employed to calculate the many body interactions of about 150,000 carbon or silicon particles with each other. We use the Brenner many-body hydro-carbon potential ${ }^{10}$ to describe the intermediate distance inter-atomic interactions, this is splined to the $\mathrm{ZBL}^{11}$ Coloumb style potential at close separations. We use an adapted Brenner potential ${ }^{12}$ to describe the long range interactions in HOPG targets, so that the graphite modelled exhibits inter-layer bonding and allows molecules to become bound in a Van der Waals fashion to the modelled graphite surface. The computation employs periodic boundary condition in the lateral dimensions ( $\mathrm{x}$ and $\mathrm{z}$ ) and free boundaries in the direction normal to the surface (y).

\section{Fullerene Scattering from Graphite Surfaces}

Ion scattering spectroscopy has been used over a number of years, normally to investigate the properties of surfaces. Typically mono-energetic ions are scattered from a surface and the reflected ions are detected and their angular and energy distribution measured. Scattering of molecular species from solids can also give some information about the strength and mechanical properties of the scattered molecule. 

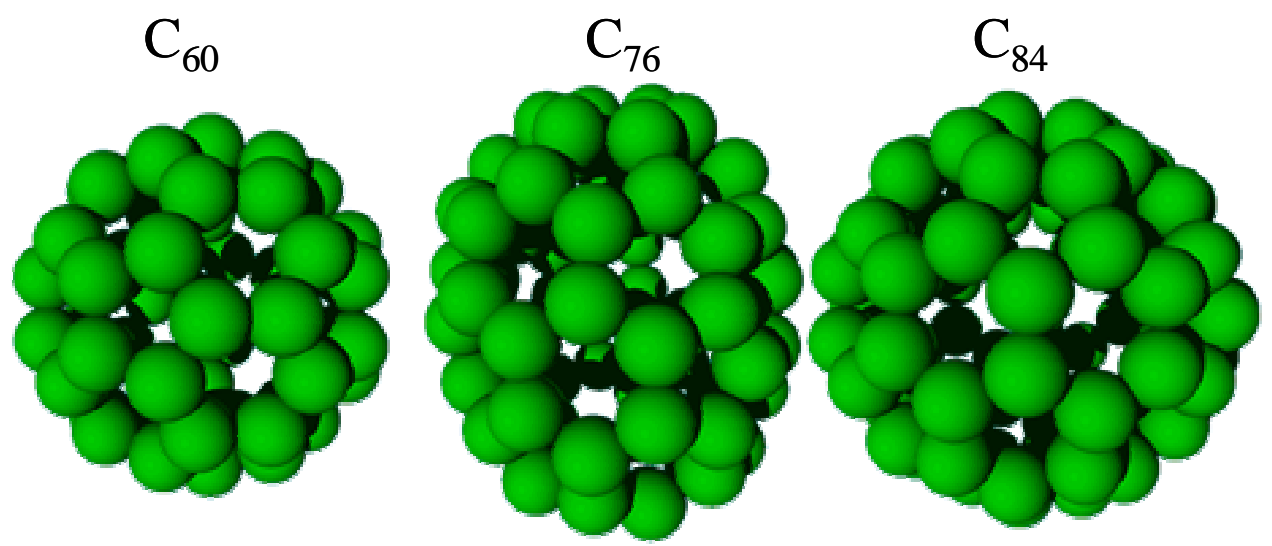

FIGURE 1. Pictorial representation of the atoms of various fullerene molecules. Note the spherical symmetry of $\mathrm{C}_{60}$ and $\mathrm{C}_{84}$ and the elongated shape of $\mathrm{C}_{76}$.

Experiments have been considered to determine whether the shape of the molecule might affect its scattering behaviour. Figure 1 shows the equilibrium positions of the atoms in three different fullerene molecules. These are the commonly know $\mathrm{C}_{60}$ molecule, as well as a $\mathrm{C}_{76}$ and $\mathrm{C}_{84}$ molecule. All three molecules result in a typical fullerene closed cage structure. Both the $\mathrm{C}_{60}$ and $\mathrm{C}_{84}$ are spherical in shape. The $\mathrm{C}_{76}$ molecule shows a more pronounced elliptical shape. From our knowledge of "sports" we are quite familiar with the different behaviour of bouncing a soccer ball and an American football on the ground. The question is "will a scattering molecule behave in a similar way?”

A series of experiments were performed ${ }^{4}$ to verify the simulation code. In these experiments $\mathrm{He}$, Xe and $\mathrm{C}_{60}$ ions were scattered from a graphite surface. Very good agreement between the experiment and simulations were found ${ }^{4}$. A number of interesting "side-effects" were identified. In particular it was noted that Xe scattered from the surface of the graphite as a result of a combination of up to 7 collisions with surface atoms. It was also noted that unexpectedly the $\mathrm{C}_{60}$ did not scatter in a specular direction as might be expected for a spherical molecule. Instead the scattered molecule was found to bounce from the surface substantially below the specular direction. Figure 2 below shows a comparison of the experimental and simulation results. The molecule is sent into the surface at $75^{\circ}$ to the surface normal $\left(15^{\circ}\right.$ to the surface $)$ so that specular reflection would be expected at $30^{\circ}$. Note how as the energy of the impact increases the scattering moves further away from specular. 


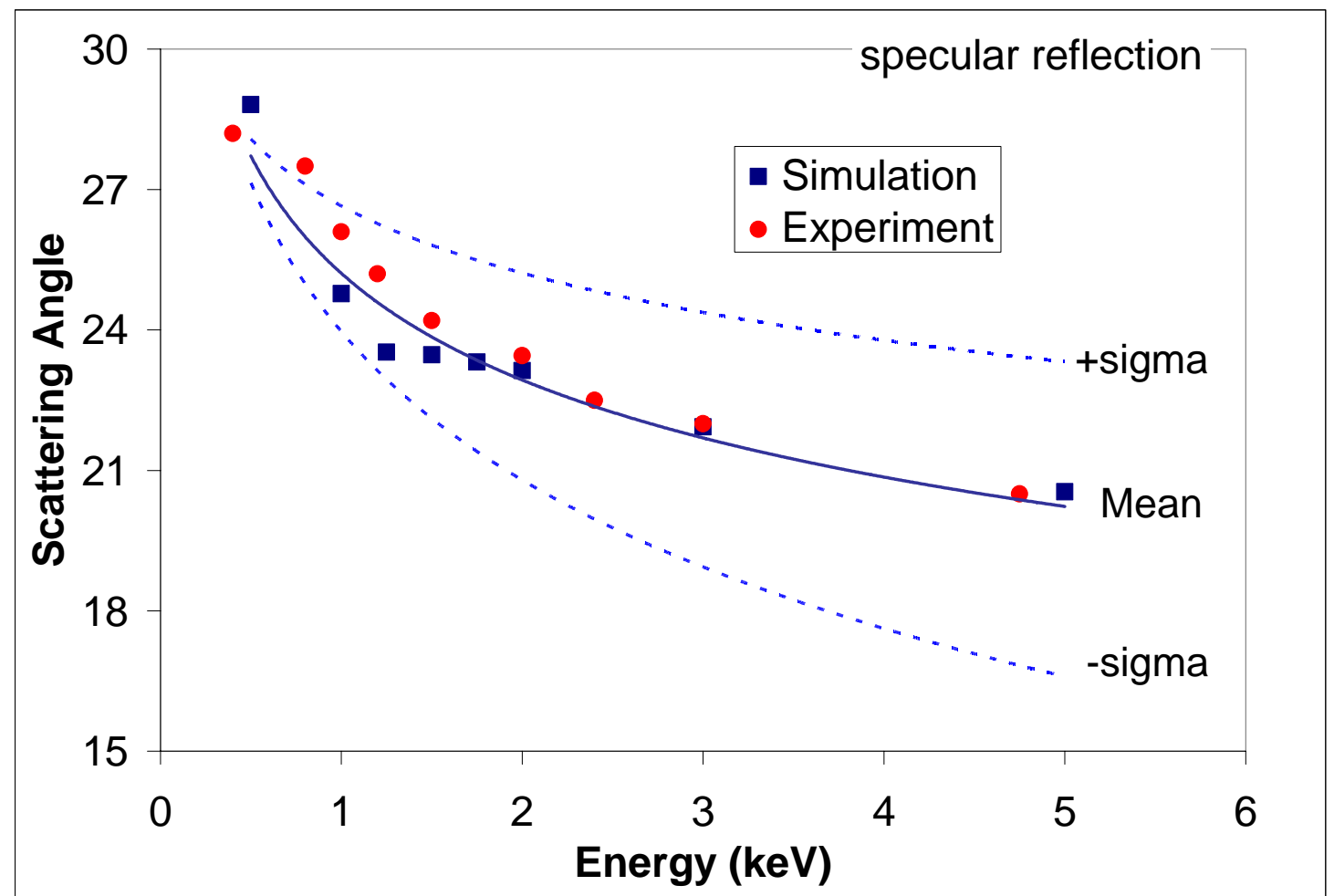

FIGURE 2. Comparison between experimental and simulated scattering of $\mathrm{C}_{60}$ fullerene molecules from a graphite surface ${ }^{4}$.

Also indicated in the figure is the width of the distribution. This is also seen to increase as the initial energy of molecule increases. The simulations here provide a useful insight into what causes this "unexpected" non-specular effect. Figure 8 below shows a visualization of the scattering events for both $\mathrm{C}_{60}$ and Xe derived from the simulations. 

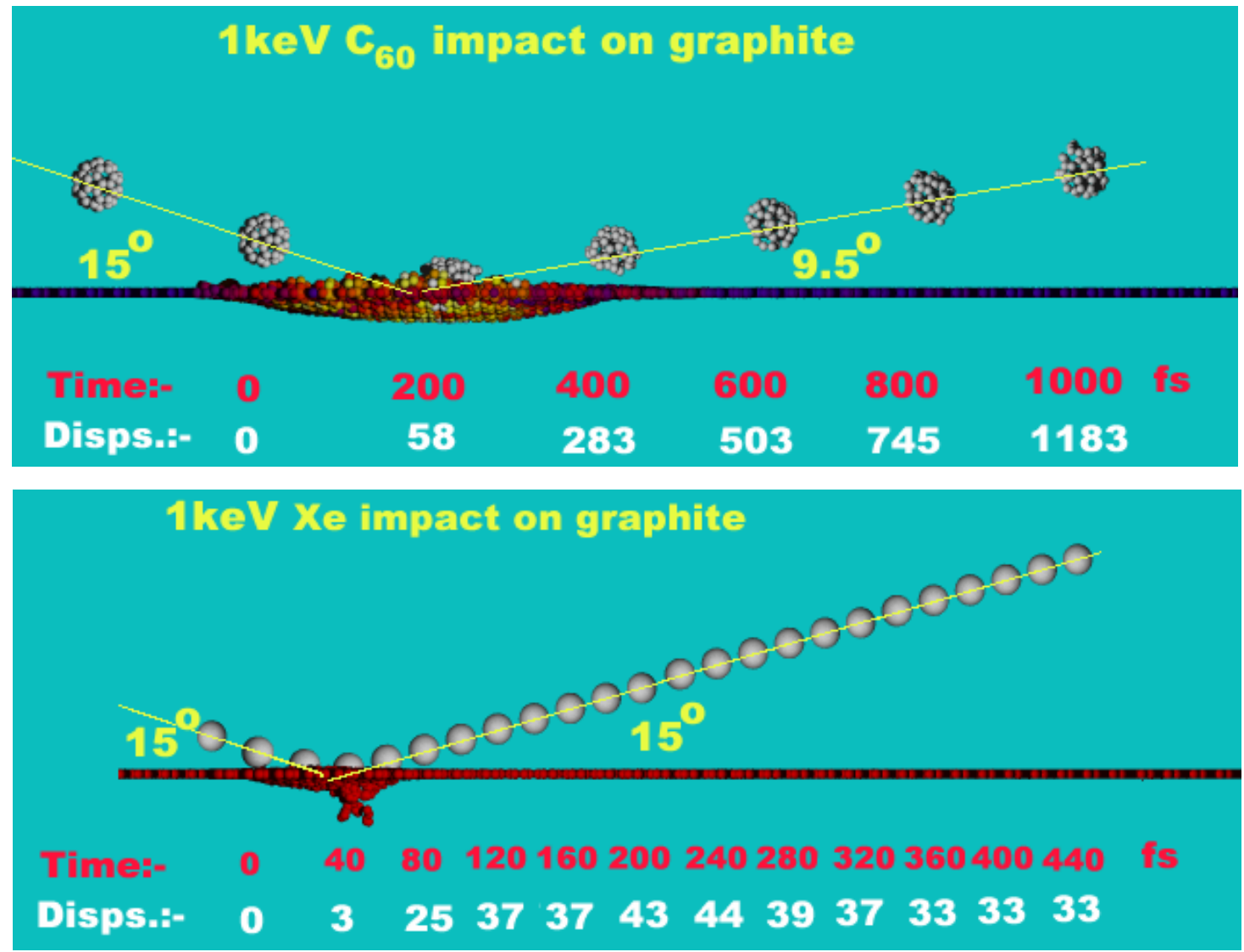

FIGURE 3. Visualisation of the scattering event for $\mathrm{C}_{60}$ and $\mathrm{Xe}$ as derived from the simulation results. Overlaid snapshots of the projectile and first surface layer atoms of the graphite surface are shown every 200 and 20 fs for $C_{60}$ and Xe respectively. The lower row in each picture lists the number of surface atoms displaced from their equilibrium positions at each time ${ }^{4}$.

From the figure it can be seen that the $\mathrm{C}_{60}$ causes substantial deformation of the surface and the whole scattering process is severely in-elastic. The $\mathrm{C}_{60}$ itself picks up a substantial amount of internal energy - often leading to fragmentation in the gas phase above the target. But it is the energy lost to deforming the target which inevitably leads to the non-specular scattering. A large part of the problem here is that graphite is made up from a set of almost uncoupled planes. These planes easily deform like an elastic membrane, as the scattering process is over quickly the surface is still distorting after the molecule has scattered. This means that energy is lost to the surface in deforming the layer on the inward path and it does not get returned to the molecule on the outward path as the deformation is still continuing. In the case of the Xe the surface deformation is relatively minor so that any loss of energy from the ion to the surface in creating the deformation is small in comparison to the overall scattering energy. Scattering simulations of $\mathrm{C}_{60}$ performed on diamond substrates show a much more specular reflection. The problem with the diamond scattering is that the fullerene molecule invariably shatters on impact leaving only scattered fragments.

The question still remains "is there any evidence that molecule shape will influence the scattering process?” 
Simulations were performed ${ }^{13}$ for an incidence angle of $80^{\circ}$ to the surface normal to a multi-layer graphite surface for a range of energies for the three fullerene molecules $\mathrm{C}_{60}, \mathrm{C}_{76}$ and $\mathrm{C}_{84}$.

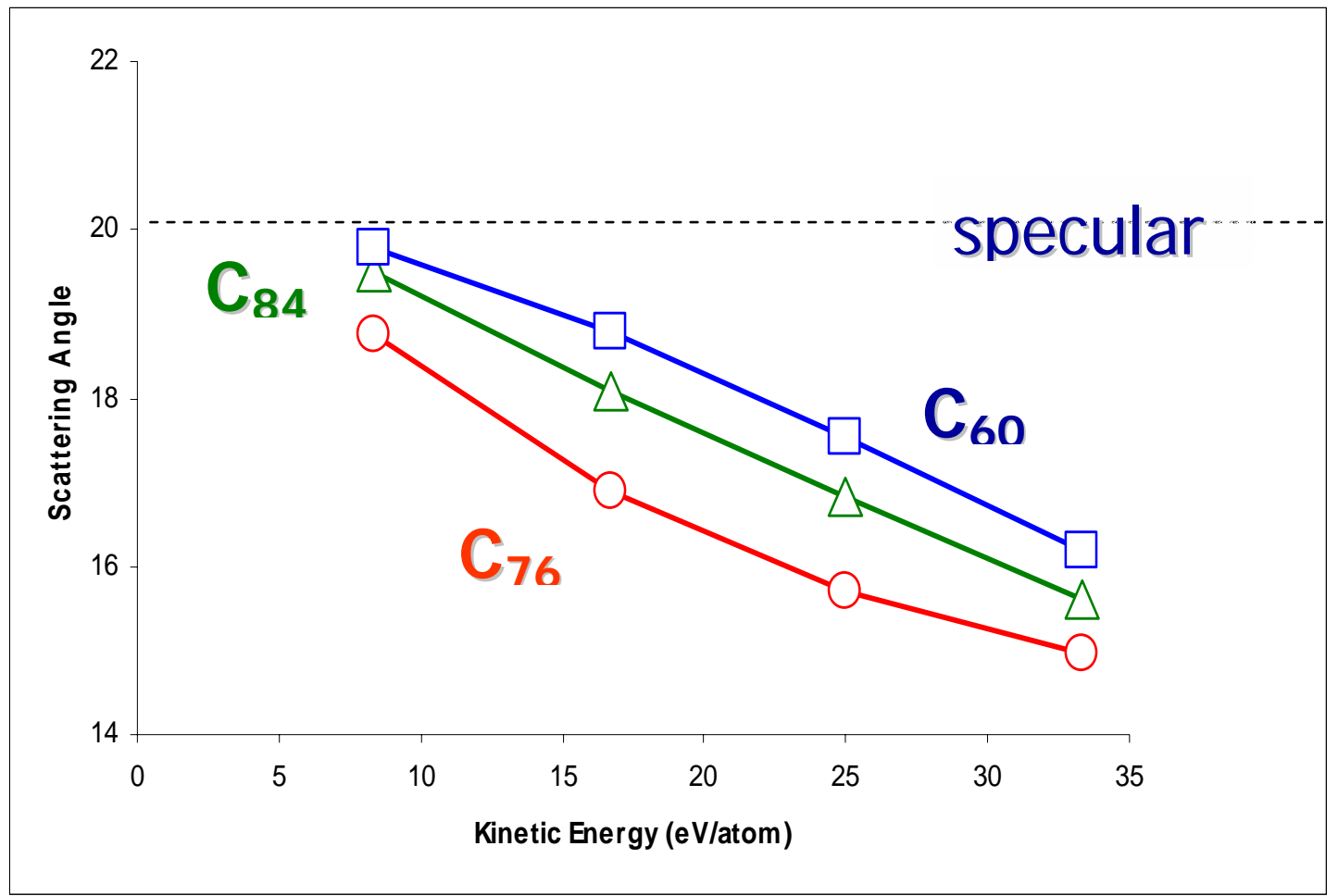

FIGURE 4. Mean total scattering angle as a function of energy for different fullerenes ${ }^{13}$.

The mean scattering angles determined as a function of initial impact energy are shown in figure 4 . The trends are much the same as discussed above for the $75^{\circ}$ incidence. That is the scattering angle becomes closer to specular the lower the energy of the impact. The thing to note is that the $\mathrm{C}_{60}$ scatters closest to specular all of the time and the $\mathrm{C}_{84}$ is next closest to specular. The $\mathrm{C}_{76}$ results are always furthest away. It might be expected that the greater the number of atoms in the fullerene the larger the deformation of the surface and hence the more the scattering angle will be nonspecular. It is quite clear that the $\mathrm{C}_{76}$ does not fit this explanation. Is this "anomalous" scattering caused by the different shape of the $\mathrm{C}_{76}$ molecule?

To answer this question simulations were performed for a series of impacts on graphite and the partitioning of the internal energy was investigated ${ }^{13}$. The partitioning of internal energy after scattering into translational, rotational and vibrational modes was investigated as a function of impact energy - see figure 5.

To compare the different fullerene surface interactions we have to be careful to take account of the different numbers of atoms in the molecules. First of all, many effects, such as the depth of penetration ${ }^{14}$ of the atoms of the fullerene scale with the velocity of the molecules. The velocity of the molecules will be the same if the kinetic energy per atom of the molecules is the same. Hence in what follows we compare fullerenes with the same initial kinetic energy per atom. 


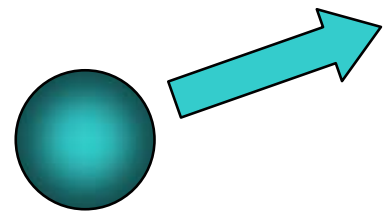

Translational

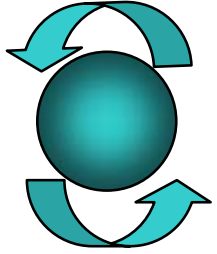

Rotational

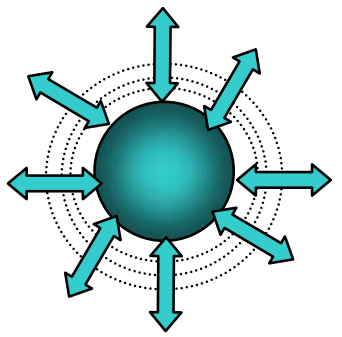

Vibrational

FIGURE 5. Different internal energy partitioning investigated by simulation after scattering fullerene molecules from a graphite surface.

This will mean that the larger fullerenes will have a larger total energy of impact. It follows from this that they can create a greater number of displacements in the substrate which normally scales with the number of atoms in the fullerene. ${ }^{5}$

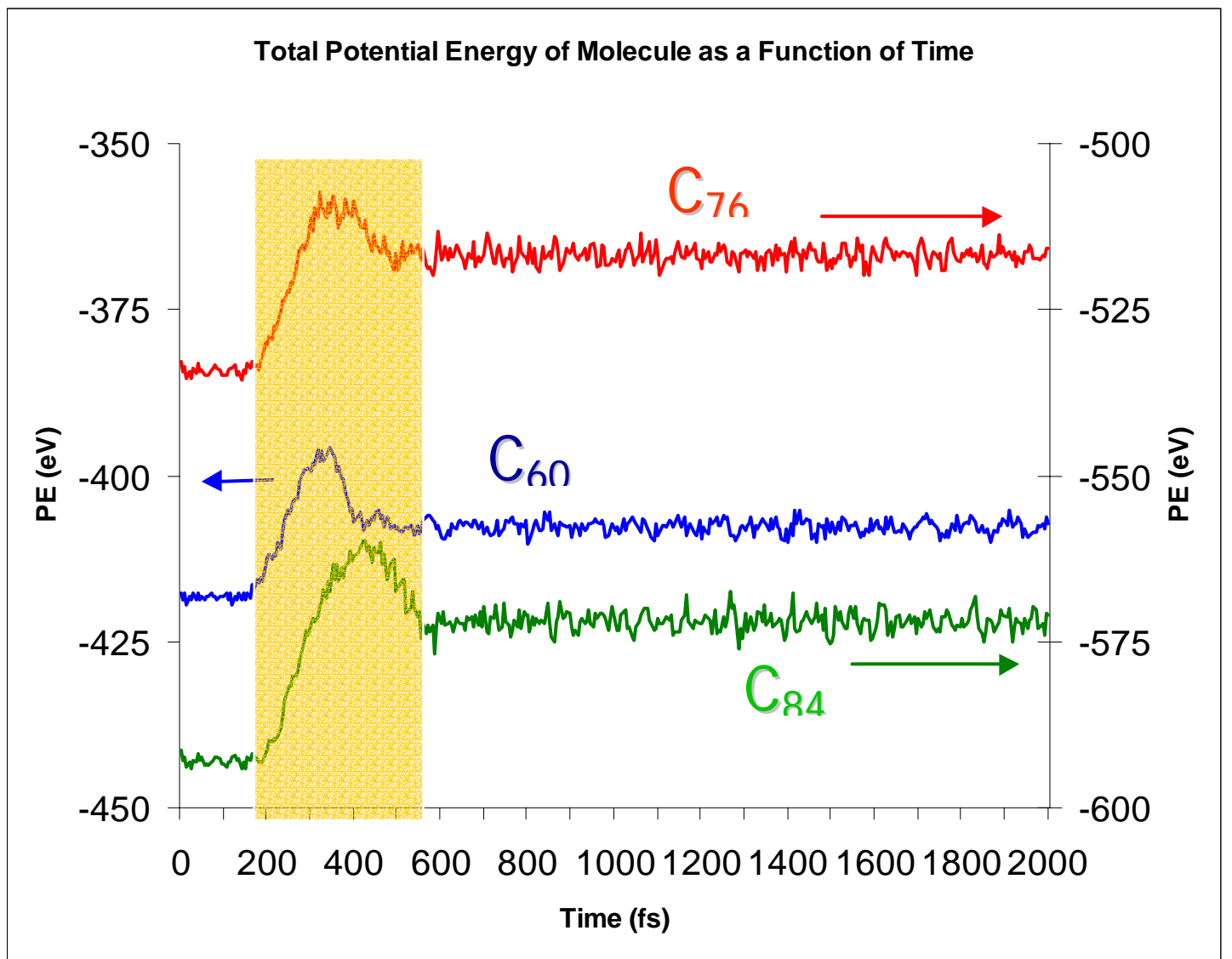

FIGURE 6. The total potential energy of the fullerene molecules as a function of time during the scattering process. The peak in the trace is caused by the interaction of the molecule with the surface.

Even though the velocity of the fullerenes is set to be the same the time during which the molecule interacts with the surface is longer the larger the molecule. Figure 6 demonstrates this by showing the total potential energy of the fullerene as a function of time during the scattering process. 
Initially the potential energy is characteristic of the fullerene with all its atoms at their equilibrium positions. As the molecule approaches the surface the potential energy increases as the molecule is compressed. Eventually the potential starts to decrease as the molecule scatters from the surface at which point the potential remains above the minimum state indicating that the molecule has acquired internal energy. Comparing the different fullerene scattering events shown in figure 6 it can be seen that the larger fullerenes interact with the surface for a longer time. The main reason for this is that they have a larger diameter. Hence they will "hit" the surface when their centres of mass are further away and continue to be attracted to the surface while their centres of mass are further away. We might consider that this is a size effect, rather than a shape effect.

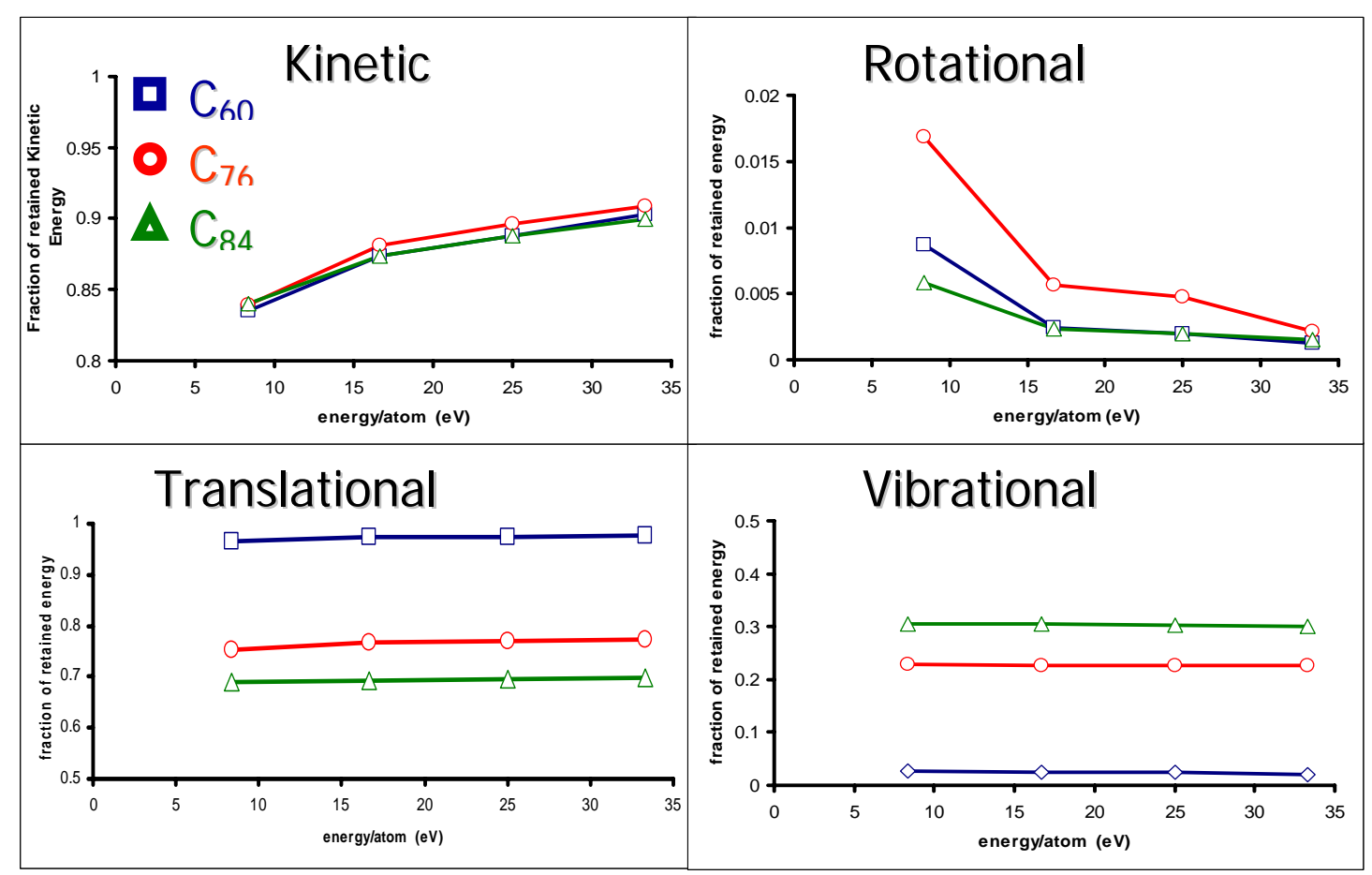

FIGURE 7. Internal kinetic energy partitioning after the scattering event as a function of impact energy for the different fullerene molecules. "Kinetic" is the fraction of the total retained kinetic energy after the impact. "Rotational" is the fraction of the retained kinetic energy that is in rotational motion of the molecule. "Vibrational" is the fraction of the retained kinetic energy that is in vibrational motion. "Translational" is the fraction of retained kinetic energy that is in translational motion of the molecule.

In figure 7 the total kinetic energy after the scattering event of the molecule is shown as a fraction of the total initial kinetic energy before the impact. This is plotted as a function of the initial impact energy for the three different fullerenes. Also plotted in the figure are the relative partitioning into rotational, translational and vibrational motion. Clearly, as the energy decreases the fraction of kinetic energy retained by the molecule decreases. All three molecules behave in a very similar way, except that the $\mathrm{C}_{76}$ appears to retain slightly more kinetic energy after scattering than the more symmetric molecules. From the same figure it is clear that the contribution to vibrational and translational motion remains static in the energy range considered and 
the fraction going to each is a function of the number of atoms (degrees of freedom) of the molecule. The only anomalous result is from the energy put into rotational motion, which is higher for the non-symmetric molecule. This appears to be due to the nonspherical shape of the molecule. There is also some evidence ${ }^{13}$ that the rotational frequency of the scattered molecule is higher in this case also.

The answer to the question about using scattering to observe shape effects would appear to be that it could be useful. There is definitely a small change in the take up of internal energy, but this might well be too small to observe experimentally. A much better parameter would be to look at the scattering angle distribution.

\section{Molecular Implantation}

As silicon chips become even faster, ultra shallow junctions at depths of less than $100 \mathrm{~nm}$ will be required ${ }^{15}$. To implant $\mathrm{B}$ atoms to these depths by conventional ion implantation is quite difficult. The acceleration voltage of the B ions can be $<1 \mathrm{keV}$ and at these voltages, space-charge blow up of the beam means that high beam currents, which are normally used in batch processing, can not be transported. This has led to the development of deceleration techniques where ion beams are extracted, mass analysed and transported at relatively high energy then decelerated before implanting into silicon ${ }^{16}$. As the deceleration stage must be very close to the target wafer, this prevents the use of batch processing of many wafers simultaneously. To provide these shallow doping layers using this technique requires the purchase of a new separate implanter.

For many years molecular ions, in particular $\mathrm{BF}_{2}$, have been used to transport low velocity boron at higher kinetic energies, thus enabling conventional implanters to be used with only minor modification to the ion source. Recently an alternative approach has been proposed using boron molecules and clusters ${ }^{17}$. If the cluster were to contain $n$ boron atoms, the added advantage is that only one charge per cluster is required to accelerate $n$ boron atoms. In addition and particularly for shallow junction applications, clusters can be transported at a relatively high energy with the result of low impact energy per boron atom. Space charge blow up of the beam can therefore be minimised and more $\mathrm{B}$ atoms implanted for the same beam current. Another potential advantage of using clusters is that the energy deposition in the crystal will tend to be localised near the surface with less channelling by individual B ions. Such channelling can occur even at low energies ${ }^{18}$. The use of decaborane - see figure 8 and also boron difluoride $\mathrm{BF}_{2}$ has been proposed as a possible means to implant $\mathrm{B}$ close to a silicon surface ${ }^{19}$. The decaborane molecule is a member of a family of around thirty boron hydrides ${ }^{20}$ but the advantage of this particular B-H cluster is that it contains a relatively large number (10) of $\mathrm{B}$ atoms and it is also less toxic than some other $\mathrm{B}_{\mathrm{x}} \mathrm{H}_{\mathrm{y}}$ compounds such as diborane or pentaborane.

Boron difluoride $\mathrm{BF}_{2}$ does not have the advantage that ten $\mathrm{B}$ atoms can be implanted for the price of one electronic charge but a possible asset is that the $\mathrm{F}$ atoms are much heavier and more reactive than the $\mathrm{H}$. Thus it might be expected that during the implantation process the $\mathrm{F}$ atoms would form a chemically altered layer above the implanted B which might be easily removed after implantation. 


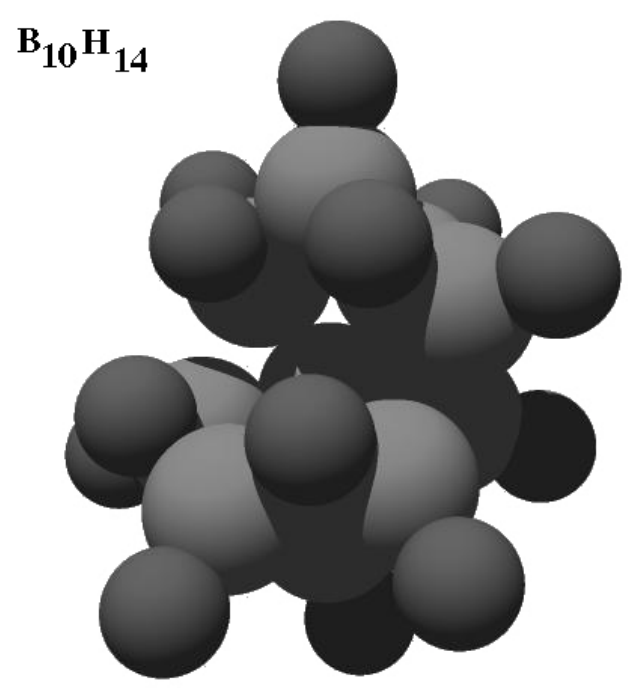

FIGURE 8. Equilibrium structure of the decaborane molecule. The larger spheres represent the boron atoms and the smaller spheres the hydrogen.

For decaborane, the short-ranged $\mathrm{Si}-\mathrm{H}$ interactions were modelled using the ZBL screened Coulomb potential ${ }^{11}$, joined smoothly to the Murty-Atwater potential ${ }^{21,22}$. The Murty-Atwater potential is a modification to the Si Tersoff potential ${ }^{23}$ which models well many of the small Si-H cluster and surface properties. There is also some agreement with ab-initio calculations of $\mathrm{H}$ interstitials but generally the short-ranged nature of the $\mathrm{Si}-\mathrm{H}$ potential which cuts off at $0.2 \mathrm{~nm}$ oversimplifies some of this description. To obtain a good description of the $\mathrm{B}-\mathrm{H}$ and $\mathrm{B}-\mathrm{Si}$ interactions is more problematical. The $\mathrm{B}-\mathrm{Si}$ interactions assumes that the $\mathrm{ZBL}$ potential is joined to a pair-wise Morse potential which has a minimum at the nearest neighbour spacing of the $\mathrm{Si}$ atoms in the lattice and a dimer binding energy of $0.25 \mathrm{eV}$. The effect is to make the substitutional site in Si a favourable position but with a relatively weak bonding of around $1 \mathrm{eV}$.

The B-B and B-H interactions are also assumed to be entirely repulsive and modelled by the ZBL potential. However in this case the potential is assumed to be very short-ranged, cutting off at $0.116 \mathrm{~nm}$ for the $\mathrm{B}-\mathrm{H}$ and at $0.15 \mathrm{~nm}$ for $\mathrm{B}-\mathrm{B}$. This is a weakness of the model for low energy interactions because it means that there is no interaction between any of the atoms in the decaborane cluster which is therefore destroyed completely on impact with the surface with no fragmentation into smaller clusters possible.

Figure 9, below, shows the implantation profiles for a set of implantation trajectories. All three profiles are similar to one another, within the statistics of the calculations. There is some evidence that the decaborane implants might be deeper on average than either the $\mathrm{BF}_{2}$ or boron implants. In general the molecular implants seem to penetrate more deeply. This is interesting in that one of the reasons proposed for using molecules was that the heavy clusters will create substantial damage in the target surface and should prevent channelling. From these results it seems that the decaborane and $\mathrm{BF}_{2}$ allows the boron to penetrate more deeply into the silicon perhaps by moving aside the host atoms to allow the boron to penetrate further. This is also 
borne out by the sticking probabilities of the different species. In the case of boron $67.5 \%$ remains within the silicon after implantation and with $\mathrm{BF}_{2}$ there is $72.5 \%$ remaining and with the decaborane $78 \%$.

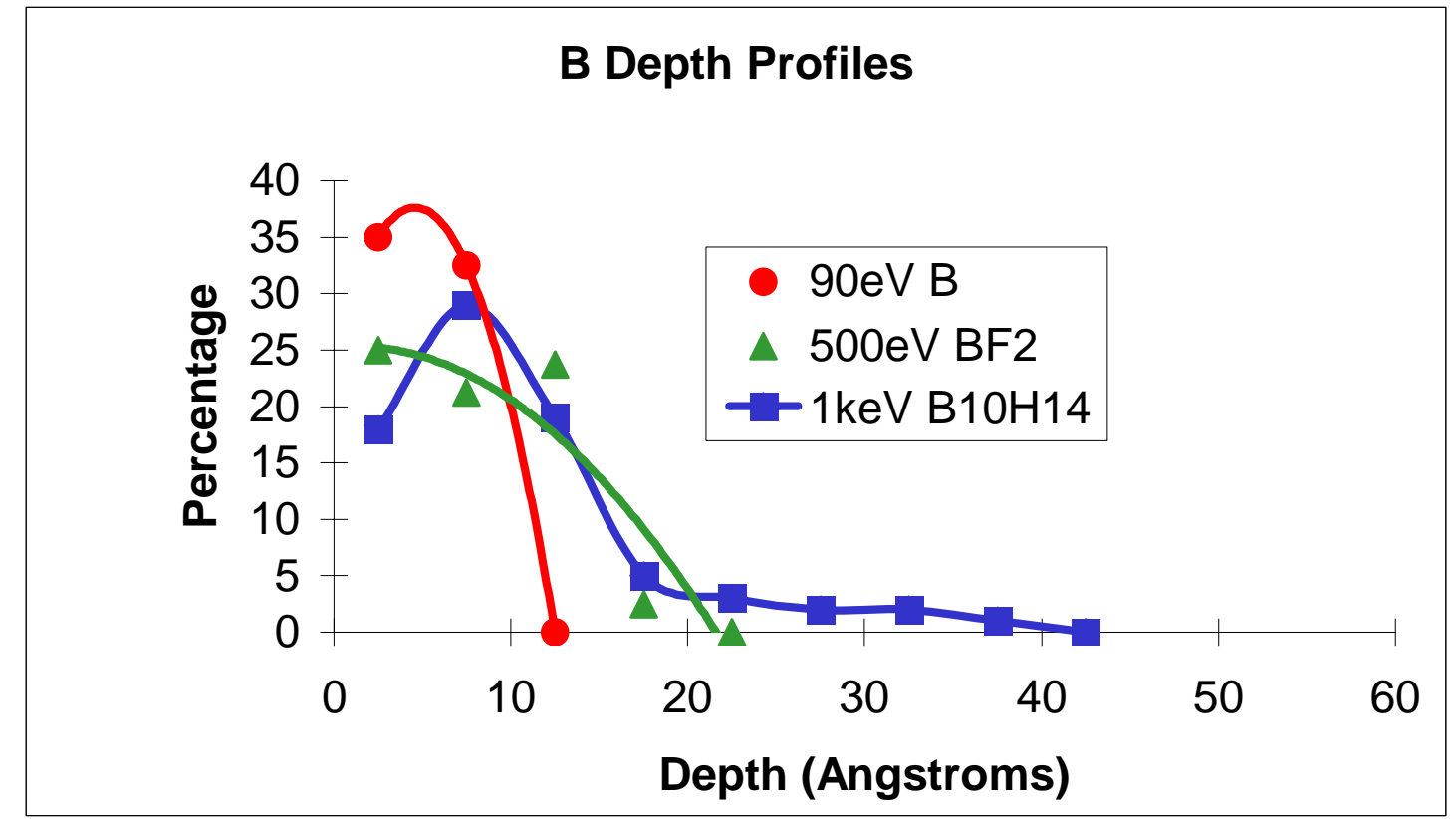

FIGURE 9. Boron depth profiles from $\mathrm{MD}$ simulations of $\mathrm{B}, \mathrm{BF}_{2}$ and decaborane implantations at the same velocity.

There is some orientation dependence of the $\mathrm{BF}_{2}$ implantation profiles. In certain orientations the fluorine in the molecule can act as a "catapult" if the molecule strikes the surface flat with the boron down. This results in an increase in the number of boron atoms reflecting from the target. When the molecule is orientated with the $\mathrm{F}$ atoms along the surface normal direction then the $\mathrm{B}$ implants more deeply (like pushing a stick into the ground).

The other important factor for the molecular implants is the question of what becomes of the hydrogen and fluorine atoms. In figure 10 we show the implantation profiles for the fluorine - in the case of the $\mathrm{BF}_{2}$ implant - and the hydrogen - in the case of the decaborane implant.

As can be seen the hydrogen penetrates more deeply into the silicon than does the fluorine. The build up of fluorine in the surface region may present a problem at these low energies and could overshadow the presence of the boron over the first half of the implantation profile. There is no sign in the time scale of the simulation (5 ps) that the fluorine is causing any etching of the surface silicon. Any silicon removed by the fluorine is via ballistic mechanisms.

Thus even with the present simplified models there is a large amount of simulations to be carried out in order fully to investigate the effects of energy and orientation on the implantation profiles and damage of the boron clusters with the silicon lattice. A fuller description of these simulations are given in refs 24, 25 and 26. 


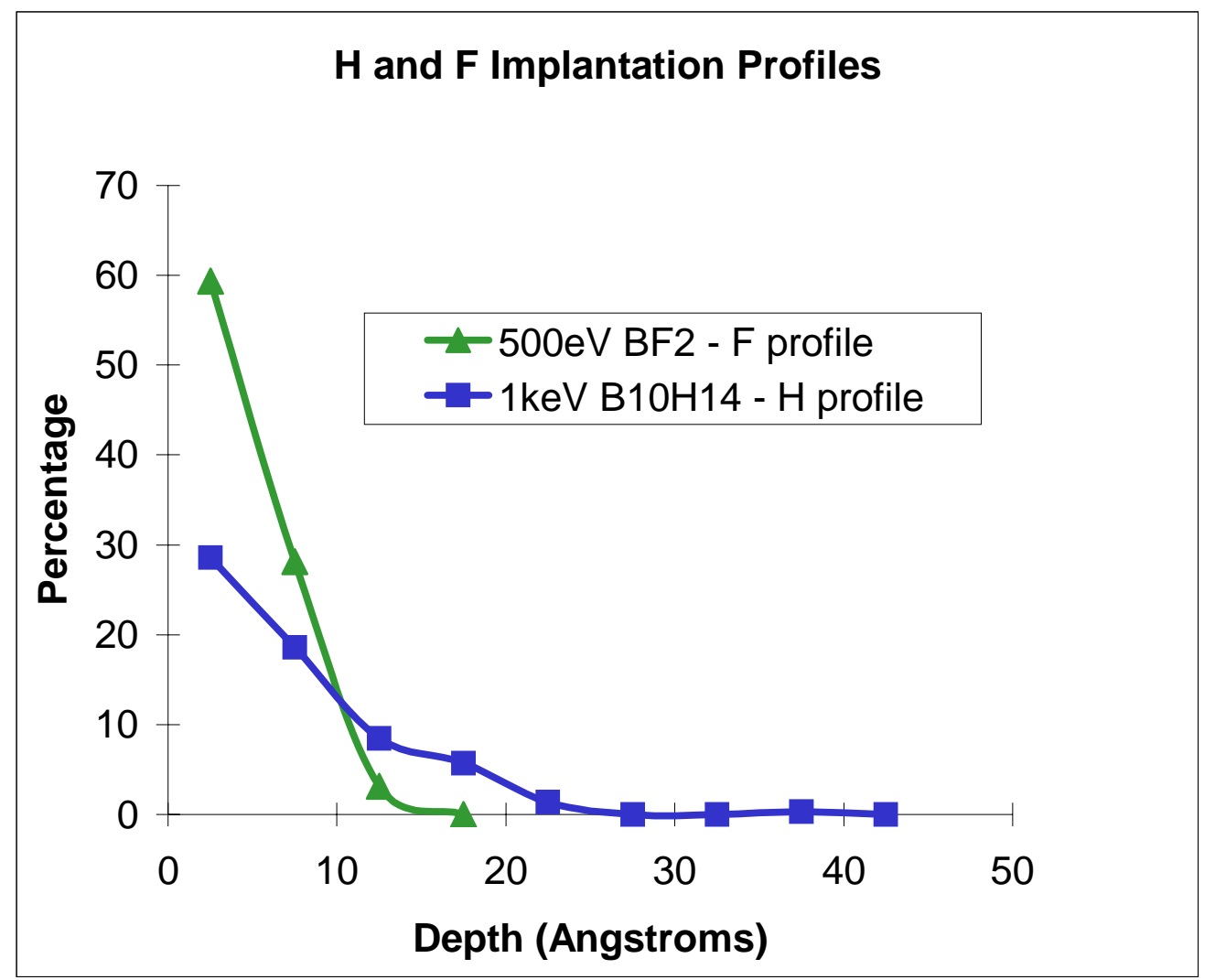

FIGURE 10. Hydrogen and Fluorine implantation profiles for decaborane and $\mathrm{BF}_{2}$ implantation in silicon respectively.

\section{Cluster Induced Desorption}

As noted in the description of the computer simulation model there is substantial interest in understanding the interactions of energetic clusters with surfaces particularly to provide understanding of the process of cluster induced desorption. One of the main areas of application of this is in cluster SIMS (Secondary Ion Mass Spectrometry). This is similar to conventional SIMS where an ion beam is used to sputter material from the surface layers of a target material, the sputtered particles are then mass analysed and detailed information about the elemental make-up of the target can be determined. With cluster-SIMS the ion beam is replaced by a cluster beam. The interest in this technique has come about as it has been found that the impact of a cluster has two very useful effects. It produces a high yield of charged particles to be sputtered from the surface - only charged particles are readily detected in SIMS providing a much improved useful yield of particles. Also the cluster impacts are more likely to sputter large organic molecules from a surface in one piece than an atomic ion beam. It has been found that conventional SIMS tends to fragment large organic molecules adsorbed on a surface making easy identification of the molecule time consuming and difficult. Using a cluster beam instead produces high yields of the large molecules and enables identification to be made more easily. 


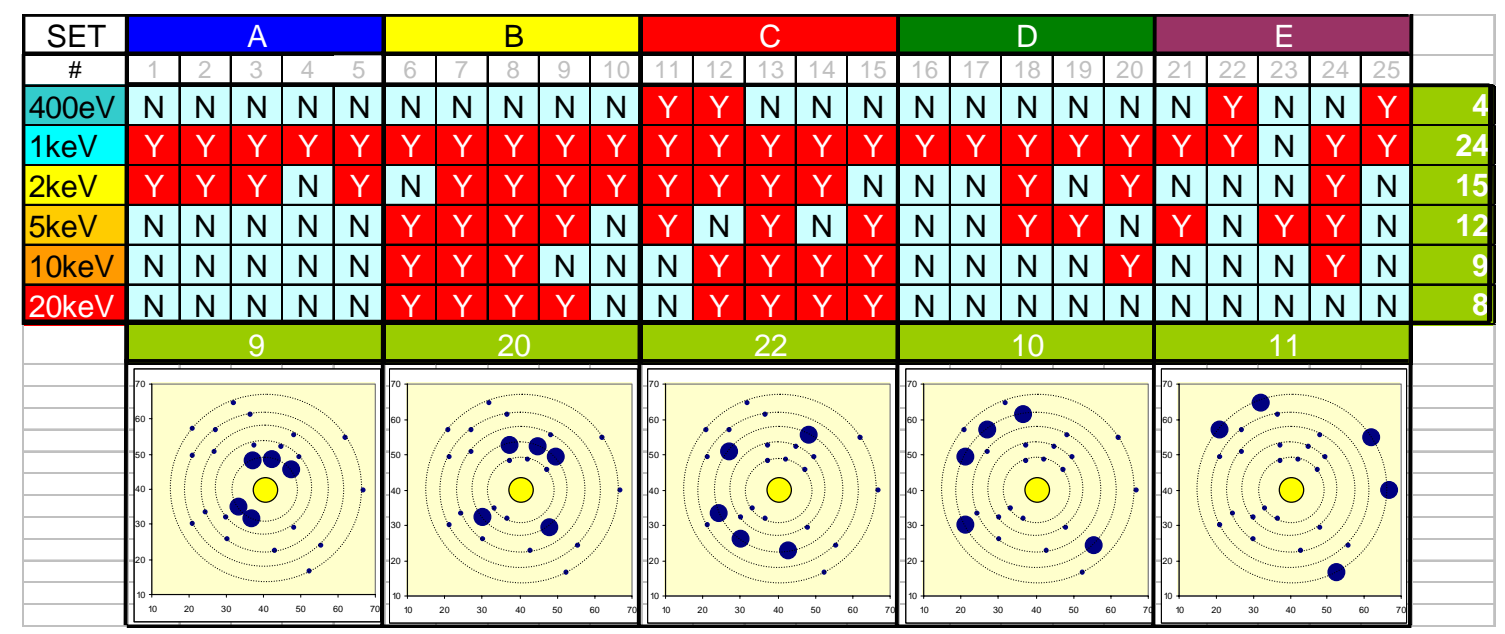

FIGURE 11. Results from an initial systematic study to determine the desorbing power of a $\mathrm{C}_{60}$ impact on a graphite surface. A set of 25 impacts have been considered in 5 rings around an initially adsorbed $\mathrm{C}_{60}$ fullerene molecule. Six different energies of impact have been investigated. In the table " $\mathrm{N}$ " indicates that the adsorbed fullerene is not desorbed after the impact and "Y" indicates that it is. The diagrams below the table show the impact points in each ring.

Figure 11, above, shows the results of an initial systematic study. The figure shows the impact points from which the initially adsorbed fullerene becomes desorbed. The desorption takes place when the impacting fullerene causes an acoustic wave to spread out from the impact site imparting enough energy to the adsorbed molecule for it to leave the surface ${ }^{27}$. One might expect that the closer the impact is to the adsorbed molecule the more likely it is to desorb it. It is reasonable to expect that the acoustic wave will lose energy as it propagates across the surface. Hence the further the wave must travel across the surface the less likely it is to cause ejection. This is borne out to some degree from these results in that there is a higher probability of ejection from impacts in the 2nd and 3rd rings than those in the 4th and 5th rings. The more interesting effect is seen with impacts in the 1st ring. At impact energies above $1 \mathrm{keV}$ none of the collisions in the first ring result in desorption of the initially adsorbed fullerene. It is reasonable to expect that if the impact is too close to the fullerene it will hit it and potentially break it, thus preventing it from escaping the surface. This does not happen here, the position of the impact was chosen specifically to prevent this from happening. The impact is far enough away that it should not directly impart energy to the initially adsorbed fullerene to desorb it. Instead what happens is that the surface around the impact site of the impacting fullerene is broken by the impact and consequently the atoms around the crater are now under co-ordinated. The consequence of this is that these atoms can become much more attractive to the adsorbed fullerene molecule and as the surface vibration interacts with the adsorbed fullerene it now brings these under co-ordinated crater atoms close. They effectively pull the adsorbed fullerene down intro the crater and more firmly attach it to the surface preventing it from leaving. A series of timeframes from a typical impact in the $1^{\text {st }}$ ring for a $5 \mathrm{keV}$ impact is shown in figure 12 to demonstrate this. 


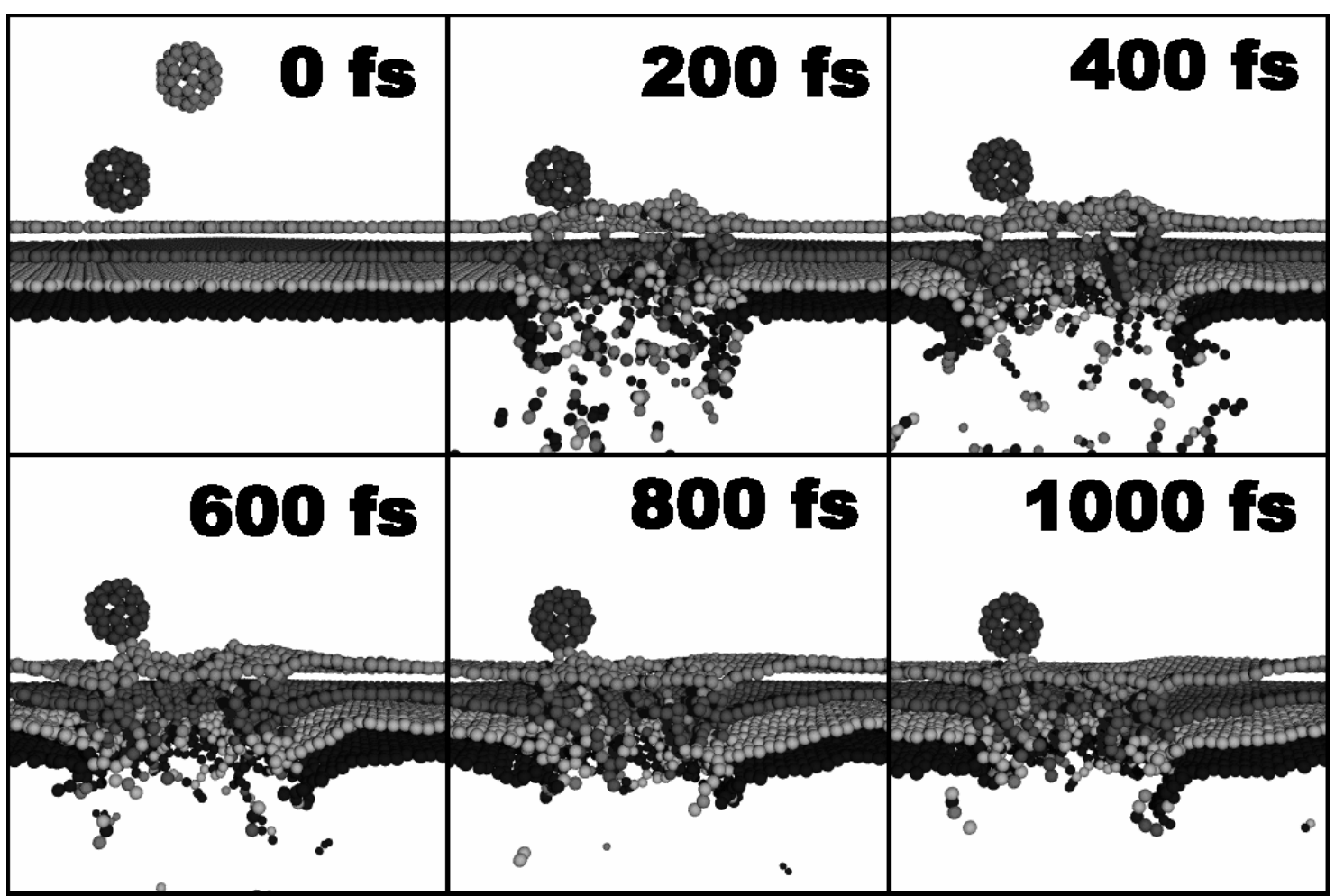

FIGURE 12. Time sequence of typical impact in ring 1 for a $5 \mathrm{keV}$ impact. Note that the initially adsorbed fullerene remains intact but attached to the graphite after the impact.

In these simulations impacts at $1 \mathrm{keV}$ are very efficient at desorbing the loosely bound adsorbed molecule. The acoustic wave created by the impact at this energy has more energy than at lower energies and can hence travel further and have a better chance of knocking off the adsorbed molecule. Impacts with energies higher than this will penetrate deeper into the surface of the graphite, create more surface damage and so not produce such a strong surface acoustic wave. It should be noted that this behaviour is very much a property of the layered structure of graphite. In a more three dimensional material such as silver or silicon this mechanism will not be so prevalent. More details on these results can be found in references 27 and 28

\section{Conclusions}

In conclusion it is hoped that this paper has demonstrated some of the interesting information that can be found from a study of molecule surface interactions. In particular it should be remembered that the value of computer simulations lays in their ability to provide insight to experimental anomalies and to provide an element of confidence to interpretation of measurements. The work presented here is only a fraction of that available in the literature and has very much been biased towards the authors own experiences and work, there is a great wealth of other material out there that has been skipped for brevities sake. 


\section{REFERENCES}

1. for example see the reviews: Harrison D.E. Jr., CRC Critical Reviews in Solid State \& Materials Science, 14(1), 1988, S1. and Harrison D.E. Jr., Radiation Effects, 70, 1983, 1.

2. Smith R. and Webb R.P., Proc. R. Soc. Lond. A 441, 1993, 495.

3. Webb R.P., Smith R., Dawnkaski E., Garrison, B. and Winograd N., Int. Vid. J. of Eng. Res. 3, 1993, 63.

4. Hillenkamp M., Pfister J., Kappes M. and Webb R.P., J. Chem. Phys. 111(22), 1999, 10303.

5. Webb R.P., Kerford M., Kappes M. and Brauchle G., Nucl. Instrum. \& Meths. B122, 1997, pp 318321.

6. Kerford M. and Webb R.P., Nucl. Instrum. \& Meths. B180, $2001,44$.

7. Webb R.P., Kerford M., Ali E., Dunn M., Knowles L., Lee K., Mistry J. and Whitefoot F., Surf. Interface Anal. 31, 2001, 297.

8. Postawa Z., Applied Surface Science, 2004 (in press).

9. Smith R., Harrison D.E. Jr., Garrison B.J., Phys. Rev. B40, 1989, 93.

10. Brenner D.W., Phys. Rev. B42, 1990, 9458. and Brenner D.W., Phys. Rev. B46, 1992, 1948.

11.Ziegler J., Biersack J. and Littmark U., The Stopping and Ranges of Ions (Pergamon, New York, 1985)

12. Smith R. and Beardmore K., Thin Solid Films, 272, 1996, 255.

13.Webb R. and Kerford M., Nucl. Instrum. \& Meths., B180, 2001, pp 32-36.

14.Webb R., Kerford M., Way A. and Wilson I.H., Nucl. Instrum. \& Meths., B153, 1999. pp 284-291.

15. National Technology Road Map for Semiconductors, Semiconductor Industry Association, San Jose, California, 2004

16. Foad M.A., England J., Moffatt S. and Armour D.G., presented at Ion Implantation Technology '96, Austin Texas, June 1996

17. Yamada I., Brown W.L., Northby J.A. and Sosnowski M., Nucl. Instrum. and Meth. B79 1993, 223

18. Smith R. and Webb R.P., Phil. Mag. Lett. 64, 1991, 253

19. Goto K., Matsuo J., Takeuchi D., Sugii T., Minikata H. and Yamada I., Application of Accelerators in Research and Industry, eds, J.L.Duggan and I.L.Morgan, AIP Press, New York, 1997 pp 937940.

20.Lipscomb W. N., Boron Hydrides, Benjamin, 1963.

21. Murty M.V.R. and Atwater H.A. Phys. Rev. B 51, 1995, 4889.

22. Beardmore K.M. and Smith R. Phil. Mag A 74, 1996, 1439.

23.Tersoff J., Phys. Rev. Lett. 56, 1986, 632; Phys. Rev. Lett. 61, 1988, 2879; Phys. Rev. B 39, 1989, 5566.

24. Smith R, Harrison M.C. and Webb R.P., Thin Solid Films, 343-344 1999 pp 602-604.

25. Smith R., Shaw M., Webb R.P. and Foad M. J.Appl. Phys. 83, 1998, 3148.

26. Webb R.P., Kerslake M., Smith R., Harrison M.C. and Foad M. Proceedings of the International Conference on Ion Implantation Technology (Eds Matsuo, Takaoka, Yamada, Published by IEEE USA), 1999 pp 764-766.

27. Webb R.P. Applied Surface Science 231-232, 2004 pp 59-63

28. Webb R.P. and Kirkby K.J., Molecular Science, in press, 2004. 